\title{
Dynamic amino acid increases in the basolateral amygdala during acquisition and expression of conditioned fear
}

\author{
B. Jill Venton, ${ }^{1}$ Terry E. Robinson, ${ }^{2}$ Robert T. Kennedy ${ }^{1,3}$ and Stephen Maren ${ }^{2}$ \\ ${ }^{1}$ Department of Chemistry, University of Michigan, 930 N. University, Ann Arbor, Ml 48109-1055, USA \\ ${ }^{2}$ Department of Psychology and Neuroscience Program, University of Michigan, 530 Church St, Ann Arbor, MI 48109-1043, USA \\ ${ }^{3}$ Department of Pharmacology, University of Michigan, 930 N. University, Ann Arbor, MI 48109-1055, USA
}

Keywords: aspartate, capillary electrophoresis, GABA, glutamate, glycine, plasticity, taurine

\begin{abstract}
Glutamate and $\gamma$-aminobutyric acid (GABA) release in the amygdala are thought to be crucial for the acquisition and expression of fear memories, but the time course of amino acid changes during conditioning is unknown. We used rapid-sampling microdialysis with $14 \mathrm{~s}$ temporal resolution to address this issue. During auditory fear conditioning, large, rapid and transient increases in glutamate and GABA were detected, but only during the first noise-shock pairing. In contrast, rats receiving unsignaled shocks during contextual fear conditioning showed no changes in GABA and less glutamate release for the initial shock, but increased glutamate release during later shocks. Expression of conditioned fear to either a white noise or the context previously paired with shock produced similar rapid and transient increases in many amino acids in the amygdala. These experiments demonstrate glutamate and GABA levels in the amygdala are differentially modulated during auditory and contextual fear learning, and are transiently increased during the expression of fear memories.
\end{abstract}

\section{Introduction}

When a neutral conditioned stimulus (CS) is paired with an aversive unconditioned stimulus (US), presentation of the CS alone comes to evoke behaviors indicative of fear. Learning associations between stimuli that predict a threatening or noxious event and that event are adaptive because they allow animals to avoid danger (Maren, 1996). CSs that elicit fear can either be discrete cues, such as a tone or light, or a complex configuration of diffuse environmental cues (termed 'context'). The neural circuits underlying both cue and context conditioning have been well studied, and the amygdala is one structure that has been implicated in the formation and storage of fear memories (LeDoux, 2000; Maren, 2001). Specifically, the basolateral complex of the amygdala receives inputs from the thalamus, cortex and hippocampus that relay sensory information, and is a critical locus for plasticity underlying the acquisition and expression of conditioned fear (Fanselow \& LeDoux, 1999; LeDoux, 2000). Pharmacological studies reveal that both $N$-methyl-D-aspartate (NMDA) and AMPA subclasses of glutamate receptors are involved in synaptic plasticity and long-term potentiation in the amygdala (Miserendino et al., 1990; Fanselow \& Kim, 1994; Walker \& Davis, 2002; Goosens \& Maren, 2004). In addition, intra-amygdala infusion of the $\gamma$-aminobutyric acid $(\mathrm{GABA})_{\mathrm{A}}$ receptor agonist muscimol before training blocks the acquisition of conditioned fear (Muller et al.,

Correspondence: Dr B.J. Venton, Department of Chemistry, University of Virginia, PO Box 400319, Charlottesville, VA 22904-4319, USA.

E-mail: bjv2n@virginia.edu

Received 11 January 2006, revised 2 March 2006, 23 March 2006, accepted 27 March 2006
1997; Wilensky et al., 1999). Therefore, glutamate and GABA neurotransmission are thought to play a critical role in the acquisition and expression of fear memories.

However, few studies have directly measured glutamate or GABA in the amygdala during fear conditioning. Microdialysis sampling has been used to demonstrate a long-lasting decrease in GABA during expression of conditioned fear (Stork et al., 2002) and a small increase in glutamate during fear conditioning (Yokoyama et al., 2005), but these studies suffer from poor (10-20 min) temporal resolution associated with traditional microdialysis. An approach that allows rapid, sub-minute sampling is required to better characterize the dynamics of neurotransmitter changes evoked by the stimuli used in fear conditioning experiments (Young, 2004).

In the present study, we use a new, high-sensitivity method coupling microdialysis sampling to capillary electrophoresis, which produces 20-80-fold improvements in temporal resolution over traditional liquid chromatography methods (Paez \& Hernandez, 2001; Parrot et al., 2003), and allows monitoring of amino acid levels with $14 \mathrm{~s}$ temporal resolution (Bowser \& Kennedy, 2001; Venton et al., 2006). The objective of this study was to determine if dynamic changes in basolateral amygdala glutamate and GABA levels occur during the acquisition and expression of conditioned fear, whether these changes differ when discrete auditory cues are used vs. more sustained contextual cues, and if plasticity in such neurochemical changes can be related to learning and memory. We found amino acid levels in the amygdala are differentially modulated during predicted shock (as in auditory conditioning) and unpredicted shock (as in contextual conditioning), and are transiently increased during the expression of fear memories. 


\section{Materials and methods}

\section{Surgery}

All animal procedures and care were approved by the University of Michigan Animal Care and Use Committee. Male Sprague-Dawley rats (Harlan, IN, USA) weighing $250-325 \mathrm{~g}$ at the start of the experiment were used. To surgically implant guide cannulae, rats were anesthetized with ketamine $(65 \mathrm{mg} / \mathrm{kg}$ i.p.) and xylazine $(15 \mathrm{mg} / \mathrm{kg}$ i.p.). Animals were placed into a stereotaxic frame and a hole was drilled into the skull for placement of the guide cannula (Plastics One, Roanoke, VA, USA) over the basolateral complex of the amygdala (coordinates in mm: AP -2.8, ML +4.9, DV -7.5). The guide cannula was fixed to the skull using surgical screws and dental cement. A second guide cannula was placed in the dental cement to use as a tether. A minimum of 5 days was allowed for the animals to recover from surgery before experiments were performed.

\section{Microdialysis with capillary electrophoresis detection}

Microdialysis probes were fashioned by ensheathing two fused silica capillaries (40 $\mu \mathrm{m}$ i.d., $105 \mu \mathrm{m}$ o.d. Polymicro Technologies, Phoenix, AZ, USA) in a cellulose acetate membrane (molecular weight cut-off 18000 Spectrum Laboratories, Rancho Dominguez, CA, USA) with a polyimide plug (Alltech, Deerfield, IL, USA). The working area of the probe was less than $2 \mathrm{~mm}$ and probe diameter was $210 \mu \mathrm{m}$. Nonactive areas of the probe were coated with polyimide. In vitro probe recoveries were about $30 \%$ for all amino acids tested when the probe was perfused at $0.8 \mu \mathrm{L} / \mathrm{min}$.

The capillary electrophoresis instrument and derivatization were similar to previously published reports (Bowser \& Kennedy, 2001). Artificial cerebral spinal fluid (in $\mathrm{mm}$ : $\mathrm{NaCl}, 145 ; \mathrm{KCl}, 2.68$; $\mathrm{MgSO}_{4} \cdot 7 \mathrm{H}_{2} \mathrm{O}, 1.0 ; \mathrm{CaCl}_{2}, 1.22 ; \mathrm{Na}_{2} \mathrm{HPO}_{4}, 1.55 ; \mathrm{NaH}_{2} \mathrm{PO}_{4} \cdot \mathrm{H}_{2} \mathrm{O}$, $0.45 ; \mathrm{pH} 7.4$ ) was perfused through the probe at $0.8 \mu \mathrm{L} / \mathrm{min}$. The microdialysate sample was mixed with $40 \mathrm{mM} o$-phthaldialdehyde, $8 \mathrm{mM} \beta$-mercaptoethanol, in $9 \mathrm{~mm}$ borate buffer with $0.8 \mathrm{~mm}$ hydroxypropyl- $\beta$-cyclodextrin to derivatize primary amines. The sample was loaded onto the separation capillary $(9.5 \mathrm{~cm}$ long, $10 \mu \mathrm{m}$ i.d.) by electrokinetic injection and the separation voltage was $-20 \mathrm{kV}$. When no injection was being made, a cross-flow buffer of $10 \mathrm{~mm}$ borate, $0.89 \mathrm{~mm}$ hydroxypropyl- $\beta$-cyclodextrin prevented leakage of the sample into the separation capillary. Laser-induced fluorescence was detected off-column in a sheath flow cuvette using a $355 \mathrm{~nm}$ diode-pumped solid state laser (DPSS Lasers, Santa Clara, CA, USA) to excite the sample. Fluorescence at $450 \mathrm{~nm}$ was collected at a photomultiplier tube orthogonally from the beam. Electropherograms were collected and analysed using in-house software written in LabView (National Instruments, Austin, TX, USA) (Shackman et al., 2004). Each test day, the system dead time (i.e. the time from sampling in the brain to detection using capillary electrophoresis) was calculated during probe calibration and confirmed by determining the delay between probe implantation and the first observed signal. The time course of the microdialysis data was corrected to account for this delay.

\section{Acquisition of conditioned fear}

All experiments were performed in a Raturn chamber (BAS, Lafayette, IN, USA), consisting of a hemispherical Plexiglas bowl (15 $\mathrm{cm}$ radius), painted black, sitting on a base that turns in the opposite direction if the animal rotates more than $90^{\circ}$ (eliminating the need for a swivel). The bottom of the bowl was fitted with parallel,
$0.64 \mathrm{~cm}$ aluminum rods (spaced $1.9 \mathrm{~cm}$ apart) that were wired to a MedAssociates (St. Albans, VT, USA) manual shocker and grid scrambler to deliver footshock. The day prior to the experiment, animals were habituated in the microdialysis testing bowl for $1 \mathrm{~h}$. On the day of the experiment, the rat was lightly anesthetized with isofluorane and the microdialysis probe was inserted through the guide cannula into the brain. A spring was used as a tether and was attached to the extra guide cannula in the dental cement. After implantation, rats were placed in a white molded plastic enclosure with a detached plastic bottom inside the microdialysis test bowl. Rats were habituated and probes equilibrated for at least $90 \mathrm{~min}$. Then at least $30 \mathrm{~min}$ of baseline samples were collected before the plastic was removed and the animal introduced into the testing environment. This context shift (upon removal of the plastic enclosure) was used to increase the animal's activity so freezing behavior during the fear conditioning trials could be assessed. Three minutes after this context shift, six conditioning trials were administered (2.5-min intertrial interval). There were three groups: a signaled shock group, which received CSUS trials; an unsignaled shock group, which received the US only; and a control group, which received no shocks but was presented the auditory CS. The CS was a 10 -s-duration, $85-\mathrm{dB}$ white noise (background white noise was $70 \mathrm{~dB}$ ) and the US was a 2-s-duration, 1-mA scrambled footshock.

Animals were videotaped for later behavioral analysis by an observer blind to treatment conditions. The behavior was assessed every $5 \mathrm{~s}$ and freezing was defined as no movements other than those necessary for respiration. The percentage of time spent freezing was then calculated for the 2-min period after the context shift and each stimulus.

\section{Expression of conditioned fear}

A separate group of animals was trained and then tested for their expression of fear to conditioned stimuli previously paired with shocks. Rats were trained for 2 days (10 daily stimulus presentations with a 1-min interstimulus interval) and then on the third day, microdialysis sampling was performed as their expression of fear to auditory and contextual stimuli was tested. Two days of training were performed to ensure robust freezing behavior when testing in a different environment; however, fear conditioning has been demonstrated with many fewer conditioning trials (Maren, 1998). All testing was performed in the Raturn testing bowl, with rats habituated in a plastic enclosure before being introduced into the testing context, as described above. There were four groups. The noise-shift group was trained with auditory CS-US presentations in a different room in standard conditioning boxes (see Maren, 1998; $30.5 \times$ $24.1 \times 21.0 \mathrm{~cm}$, with a grid floor and a fan for $70 \mathrm{~dB}$ background noise; MedAssociates). On the testing day, they were introduced into the testing context (the bowl that was never associated with shock) and then 3 min later presented the auditory CS previously paired with shock. The second group, the noise-same group, was trained in the microdialysis testing bowl with CS-US presentations. During testing, the noise-same animals were introduced back into the training context (by removing the plastic enclosure after the habituation period) and then presented an auditory CS previously paired with shock 3-min after the enclosure was removed. The context-same group was trained with only US presentations in the microdialysis testing bowl. For testing, the rats were placed in the bowl, but within the plastic enclosure described above, for the habituation period. The enclosure was then removed to introduce the rats to the training context previously paired with shock and then 3 min later a novel 
white noise was presented. The control group was trained with presentations of the white noise in the microdialysis testing bowl, but never received footshock. On the testing day, control rats were introduced back into the bowls for a habituation period in the enclosure, and then exposed to the neutral training environment by removing the enclosure and then presented with the white noise $3 \mathrm{~min}$ later. For these experiments the CS was a 30 -s-duration, $85-\mathrm{dB}$ white noise and the US was a 2-s-duration, 1-mA footshock. Behavioral reactions were assessed from videotapes every $2 \mathrm{~s}$. The percentage of time the animal froze in the 30 -s period after the context shift and during the 30-s duration noise CS was calculated.

\section{Histology}

After the experiments, rats were deeply anesthetized with sodium pentobarbital and perfused intracardially with $0.9 \%$ saline followed by $10 \%$ formaldehyde. Brains were removed and stored refrigerated in $10 \%$ formaldehyde. Coronal sections $(30 \mu \mathrm{m})$ were obtained using a cryostat, mounted onto slides, and stained with thionin. Slices were compared with a rat brain atlas to confirm probe placement (Paxinos \& Watson, 1998). Because of the length of the probes, sampling was likely from both the basolateral and lateral portions of the amygdala. However, probes were placed laterally to avoid sampling from the central nucleus of the amygdala. Animals were excluded if over half the probe length was outside the basolateral amygdala.

\section{Statistics}

Data are given as mean \pm SEM. Two-way repeated-measures ANOVAs, one-way ANOVAs and $t$-tests were calculated using GraphPad Prism. Data were considered statistically significant at the $95 \%$ confidence level.

\section{Results}

\section{Acquisition of conditioned fear}

Figure 1 illustrates microdialysis probe placements in the basolateral complex of the amygdala. Microdialysis samples were analysed online every $14 \mathrm{~s}$ using capillary electrophoresis. Six amino acids: glutamate, GABA, glycine, aspartate, taurine and serine were separated and detected. Before the stimuli were presented for the fear conditioning experiments, $30 \mathrm{~min}$ of data (130 samples) were averaged to obtain a baseline and all data are expressed as a percentage of that basal level (basal $=100 \%$ ). Basal in vivo concentrations, estimated from in vitro calibrations, were $1.6 \pm 0.2 \mu \mathrm{M}$ for glutamate, $0.10 \pm 0.02 \mu \mathrm{M}$ for GABA, $0.48 \pm$ $0.05 \mu \mathrm{M}$ for aspartate, $7.2 \pm 1.3 \mu \mathrm{M}$ for taurine, $5.7 \pm 1.6 \mu \mathrm{M}$ for glycine and $27 \pm 5 \mu \mathrm{M}$ for serine. Baseline data were collected while the animal was in a plastic enclosure before a context shift into the microdialysis testing bowl.

Figure 2 shows the time course of average changes in glutamate and GABA during acquisition of conditioned fear. Each animal was exposed to the training context at the time indicated by the open circle. Three minutes later, a series of six conditioning trials (triangles) were performed $2.5 \mathrm{~min}$ apart. In the signaled shock group (Fig. 2A), each trial consisted of a 10 -s white noise CS that co-terminated with a 2-s, $1-\mathrm{mA}$ footshock US (i.e. auditory conditioning). Animals in the unsignaled shock group (Fig. 2B) received identical, 2-s, 1-mA shocks in the absence of a discrete CS (i.e. context conditioning). Animals in

\section{$\begin{array}{lll}\text { A. Acquisition } & \text { B. Expression }\end{array}$}

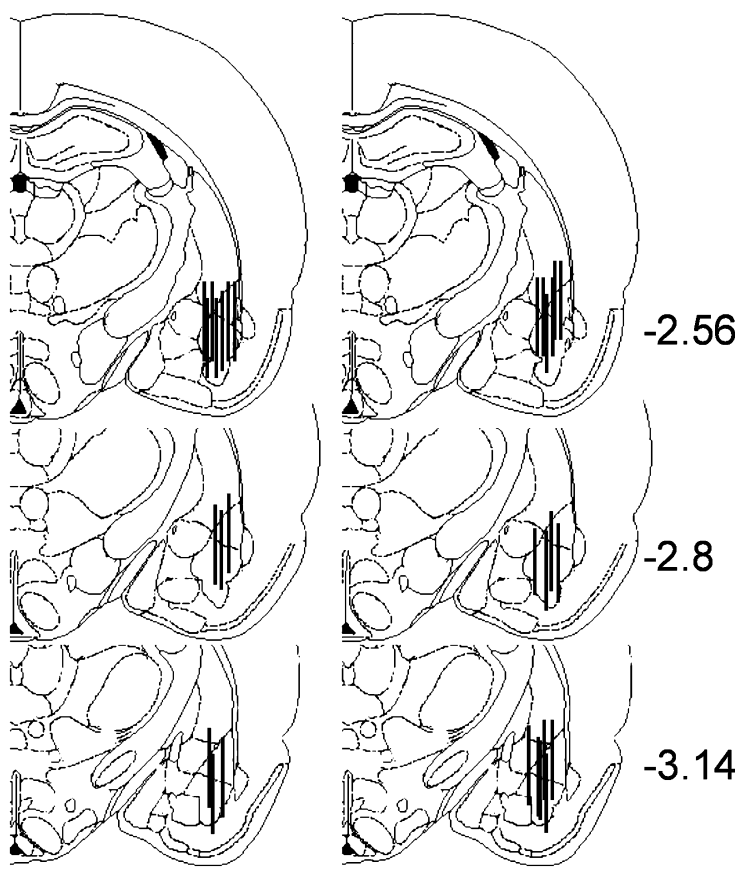

FIG. 1. Microdialysis probe placements. (A) Probe placements in the basolateral complex of the amygdala for the signaled shock, unsignaled shock and control groups for conditioned fear acquisition experiment. (B) Probe placements in the basolateral amygdala for the noise-shift, noise-same, context-same and control groups for the conditioned fear expression experiment. The numbers are $\mathrm{mm}$ from bregma.

the control group (Fig. 2C) received 10-s white noise presentations, but no shocks.

In the signaled shock group, the first noise-shock pairing produced a large increase in glutamate and GABA (Fig. 2A). The concentration rose immediately after the stimulation and the transient lasted less than $2 \mathrm{~min}$ so amino acid levels were near baseline before the next conditioning trial. However, this large amino acid response did not occur during subsequent noise-shock presentations. In contrast, the first unsignaled shock trial produced a smaller increase in glutamate release but larger, transient increases were observed for repeated trials (Fig. 2B). This response appeared relatively sustained across the conditioning session, but examination of the data from individual animals showed clear transient peaks following each shock. These transients were masked in the averaged data because the peaks did not always align perfectly in time from animal to animal. Only small increases in GABA were observed on any of the unsignaled shock trials. Finally, white noise presentations alone had no effect on glutamate and GABA levels in the control group (Fig. 2C). These control animals were administered a footshock $20 \mathrm{~min}$ after the set of white noises as a positive control (data not shown). Amino acid changes were observed in all control animals in response to the shock, verifying that their probes were functional.

To compare the pattern of responses to conditioning trials between groups, the amino acid peaks for each conditioning trial (as in Fig. 2) were integrated to obtain the area and then results from individual animals averaged (Fig. 3). The area under the curve gives an indication of both the concentration and duration of amino acid signaling. It is clear that the pattern of the amino acid response was different during the acquisition of auditory (signaled) vs. context (unsignaled) conditioning. The area under the curve is smaller for the 
A. Signaled shock

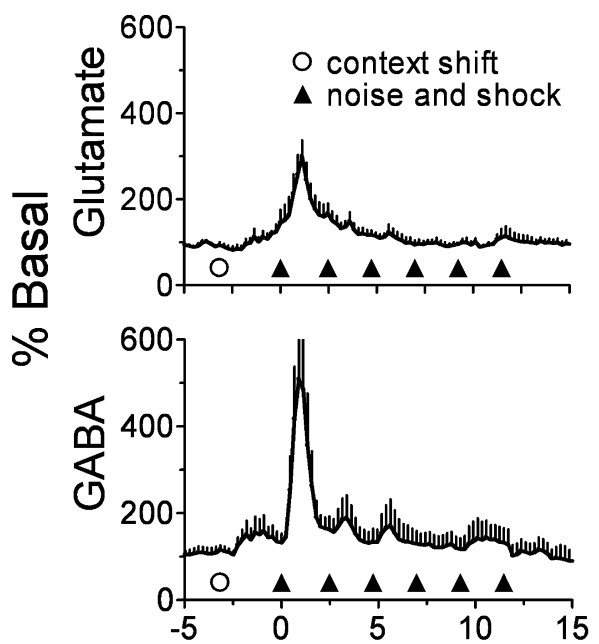

B. Unsignaled shock
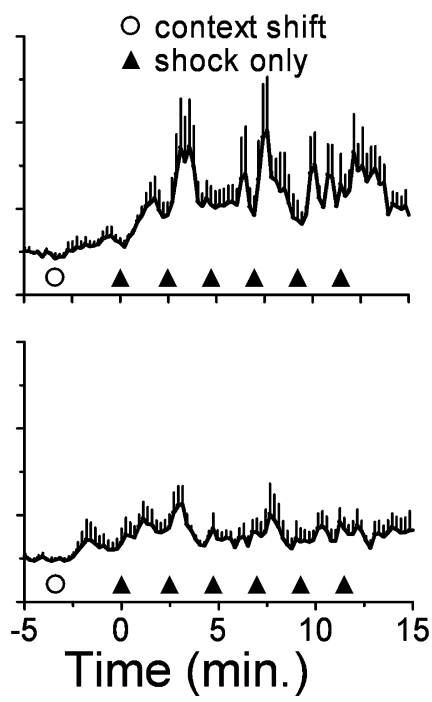

C. Control
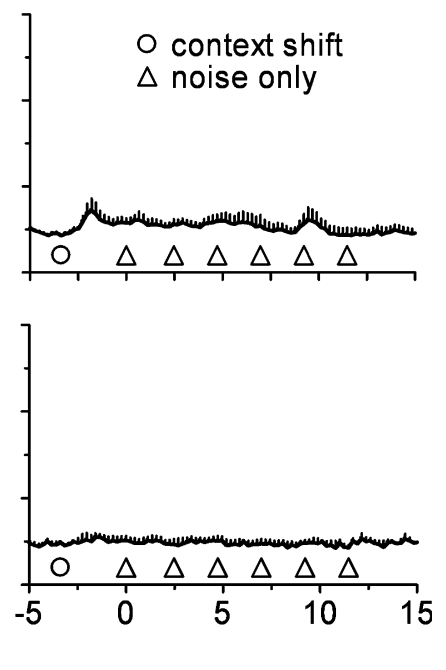

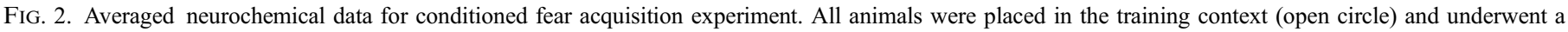

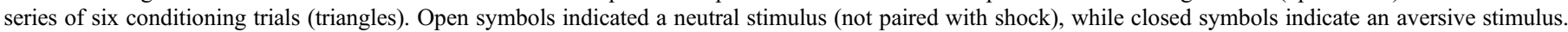

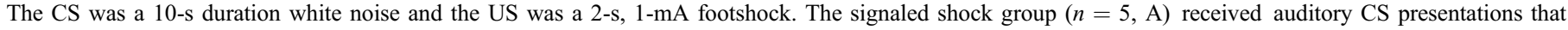

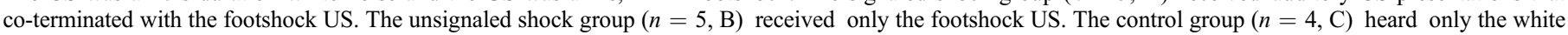

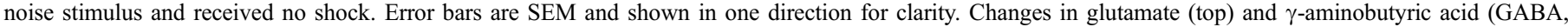

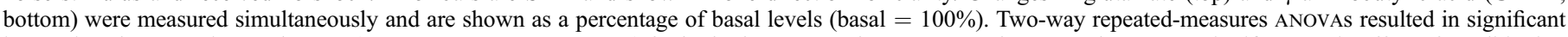

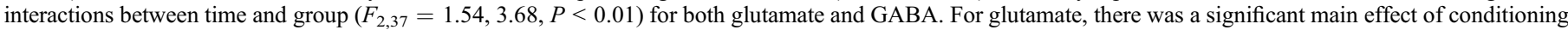

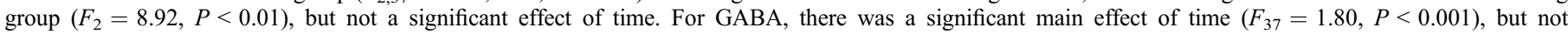
conditioning group.

\section{A. Glutamate}

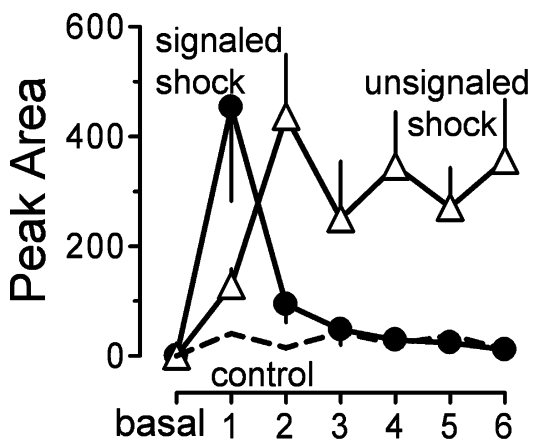

B. GABA

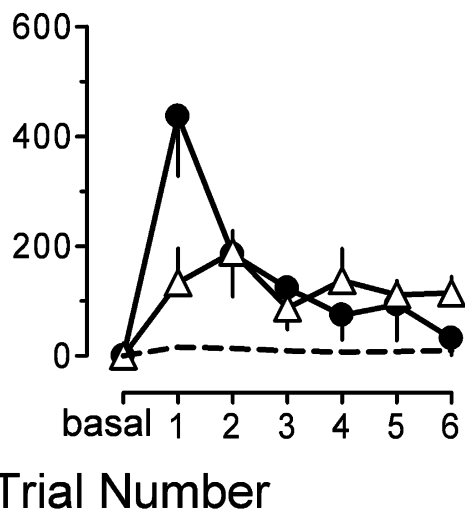

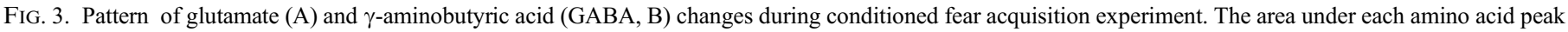

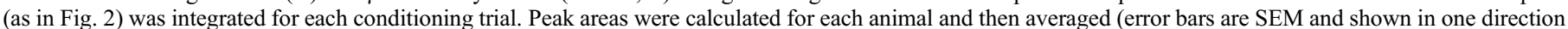

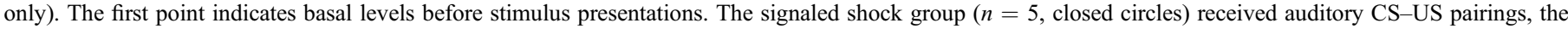

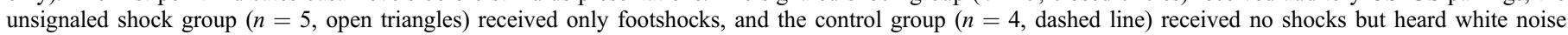
stimuli.

unsignaled shock group on the first conditioning trial for both GABA and glutamate (Bonferonni post-test after two-way ANOVA, $P<0.05$ ). During subsequent conditioning trials, glutamate release is larger in the unsignaled shock group, but GABA release is similar in both the signaled and unsignaled shock groups.

Other amino acids, including aspartate, taurine, glycine and serine, were also measured simultaneously during conditioning, and the maximal response on the first and last conditioning trials is shown in Table 1. Similar to the results for glutamate and GABA, signaled shocks elicited a large increase in aspartate, taurine and glycine on the first conditioning trial, but little release on later trials. In contrast, unsignaled shocks produced transient increases for these amino acids on all conditioning trials. Increases in serine were smaller, showing not all amino acids dramatically increased.

Freezing behavior was scored during the conditioning session and is shown in Fig. 4. The baseline freezing level was low, indicating that the animals were active at the start of the conditioning session. Animals in the signaled and unsignaled groups showed high levels of freezing after footshock. Interestingly, some of the animals receiving unsignaled shocks became agitated during the later trials and tried to 
TABLE 1. Maximal amino acid peaks for the first and sixth conditioning trial during acquisition of conditioned fear

\begin{tabular}{|c|c|c|c|c|c|c|}
\hline \multirow[b]{3}{*}{ Amino acid } & \multicolumn{6}{|c|}{ Amino acid peaks $(\%)^{\dagger}$} \\
\hline & \multicolumn{2}{|c|}{ Signaled shock } & \multicolumn{2}{|c|}{ Unsignaled shock } & \multicolumn{2}{|l|}{ Control } \\
\hline & Trial 1 & Trial 6 & Trial 1 & Trial 6 & Trial 1 & Trial 6 \\
\hline Glutamate & $400 \pm 80^{*}$ & $110 \pm 10$ & $200 \pm 40$ & $390 \pm 60 *$ & $130 \pm 20$ & $100 \pm 10$ \\
\hline GABA & $560 \pm 140^{*}$ & $140 \pm 30$ & $200 \pm 40$ & $210 \pm 30$ & $105 \pm 10$ & $106 \pm 7$ \\
\hline Aspartate & $600 \pm 150^{*}$ & $120 \pm 10$ & $220 \pm 40$ & $440 \pm 70 *$ & $130 \pm 30$ & $100 \pm 20$ \\
\hline Taurine & $480 \pm 100^{*}$ & $150 \pm 40$ & $310 \pm 100$ & $320 \pm 40^{*}$ & $110 \pm 10$ & $103 \pm 8$ \\
\hline Glycine & $500 \pm 40^{*}$ & $130 \pm 20$ & $300 \pm 100$ & $320 \pm 50^{*}$ & $110 \pm 5$ & $104 \pm 10$ \\
\hline Serine & $170 \pm 30$ & $120 \pm 20$ & $180 \pm 50$ & $150 \pm 70$ & $105 \pm 10$ & $106 \pm 7$ \\
\hline
\end{tabular}

${ }^{\dagger}$ Percentage of basal values. ${ }^{*} P<0.05$, compared with basal levels.

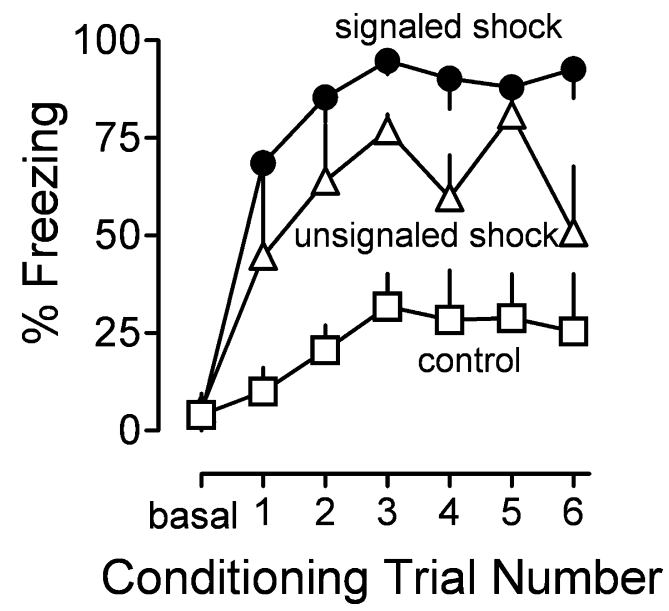

FIG. 4. Conditioned fear acquisition behavioral responses. The percentage of time animals spent freezing is plotted after the context shift (basal) and for the 2 min following each stimulus. Signaled shock animals ( $n=5$, closed circles) received auditory CS-US pairings, unsignaled shock animals $(n=5$, open triangles) received context conditioning with only shock presentations, and control animals ( $n=4$, open squares) were presented only with white noise stimuli. A two-way repeated-measures ANOVA showed a significant main effect of group $\left(F_{2}=49.5, P<0.001\right)$, but not conditioning trial number $\left(F_{5}=2.1\right.$, $P>0.05)$. One-way ANOVA tests comparing individual groups showed that freezing behavior in the signaled and unsignaled shock groups was significantly greater than control $\left(F_{1}=119,23.4, P<0.0001\right)$, and the signaled shock behavior was significantly different to unsignaled shock behavior $\left(F_{1}=18.5\right.$, $P<0.01)$. There was no significant effect of conditioning trial in any of the one-way ANOVA comparisons.

escape the bowl. A comparison of Figs 3 and 4 reveals that although the pattern of behavior was similar for the signaled and unsignaled conditioning groups, the pattern of amino acid neurotransmission differed depending on the conditioning paradigm.

\section{Expression of fear to conditioned stimuli}

The concentrations of amino acids during the expression of conditioned fear were quantified in separate groups of rats. Animals were trained with 10 conditioning trials on two consecutive days. On Day 3, microdialysis samples were collected as their expression of fear to CSs was tested (Fig. 5). The noise-shift group (Fig. 5A) was trained in a separate context with a discrete white noise CS paired with a footshock US. On the testing day, they were introduced into a novel environment (not paired with shock) and then presented the white noise previously paired with shock. This noise-shock group did not respond to the novel environment, but subsequent presentation of the white noise CS caused a rapid and transient increase in glutamate and GABA (Fig. 5A). Animals in the noise-same group (Fig. 5B) were trained in the same context used for testing with an auditory CS predicting the shock (i.e. signaled shock). During testing, they were introduced back into the training context previously paired with shock and then presented the white noise CS. In the noise-same animals, introduction to the training context produced an immediate, rapid increase in glutamate and GABA, but no separate peak in response to the white noise CS (Fig. 5B). The context-same group (Fig. 5C) was trained in the testing context without any discrete cues predicting the US (i.e. unsignaled shock). They were tested by introduction into the testing context previously paired with shock and then presented a novel white noise. In the context-same animals, similar to the noisesame animals, exposure to the training context produced a large and immediate increase in amino acids but no response to subsequent presentation of white noise (Fig. 5C). The control group (Fig. 5D) was presented white noise stimuli during training but did not receive shocks. On the testing day, the control animals had no neurochemical response to the training environment or white noise presentation.

Table 2 shows the maximal changes measured for each amino acid in response to the first stimulus previously associated with shock. For the control group that did not receive shocks, the response to the first stimulus, a context change, is shown. Similar to the acquisition experiments, multiple amino acids were released in response to cues previously paired with shock. The patterns of amino acid release were similar for glutamate, aspartate, taurine and glycine. Serine was not significantly increased in any group.

Freezing behavior during expression of conditional fear is shown in Fig. 6. The control group showed little freezing after placement in the new environment, indicating animals were normally active after the context shift. For the experimental groups, the amount of freezing to the first stimulus presentation previously paired with shock is shown. The percentage of time spent during the $30 \mathrm{~s}$ white noise previously paired with shock is illustrated for the noise-shift group. The amount of freezing for the 30 -s period after placement in the training context, previously paired with shock, is pictured for the noise-same and context-same groups.

Amino acid transients were also occasionally observed in the noiseshift, noise-same and context-same groups that were not time-locked with the presented cues. In these groups, the random transients most often occurred after the presentation of the CS previously associated with shock. The presence of transients not directly associated with the cues suggests there may be some shock-sensitization of amino acid release. Sensitization was not observed in the control group that received no footshocks and just heard white noise stimuli. 

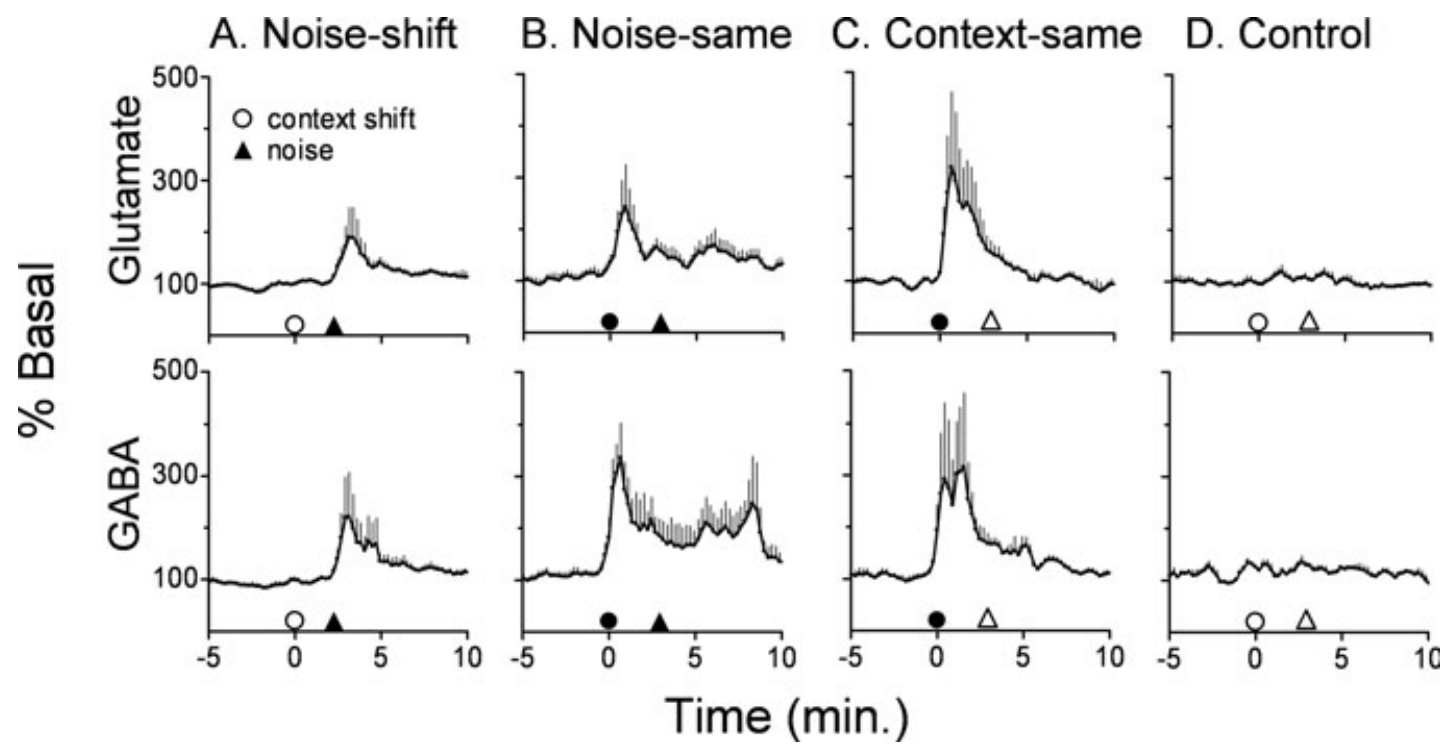

FIG. 5. Neurochemical changes during expression of conditioned fear. Four groups of rats were trained with various combinations of contextual or auditory stimuli for 2 days to promote fear conditioning. On the third day, the neurochemical responses of all rats were tested by a context shift into the microdialysis bowl (circle) followed by presentation of a white noise (triangle). Open symbols represent a neutral stimulus presentation (not previously paired with shocks), while closed symbols represent an aversive stimulus presentation previously paired with shock. The noise-shift group $(n=4, \mathrm{~A})$ was trained in a separate context with a white noise CS predicting the US. On testing day they were introduced into a novel context and then presented the auditory CS previously paired with shock. The noisesame group $(n=4, \mathrm{~B})$ was trained in the microdialysis testing bowl with noise-shock presentations and then tested by placement into the training context followed by presentation of the auditory CS previously paired with shock. The context-same group $(n=5, \mathrm{C})$ was trained in the microdialysis testing bowl with only footshocks. They were introduced back into the context paired with shock and then presented a novel white noise on the testing day. The control group $(n=4$, D) heard white noise stimuli but received no shocks during training in the microdialysis test bowl. On the testing day, they were reintroduced into the training context and then presented the white noise. Error bars are SEM and shown only in one direction. Two-way repeated-measures ANOVA analysis revealed a significant interaction for both glutamate and $\operatorname{GABA}\left(F_{3,27}=1.48,1.79, P<0.01\right)$, and a significant main effect of time $\left(F_{27}=2.98,4.20, P<0.001\right)$, but no significant main effect of group.

TABLE 2. Maximal percentage of basal changes for expression of conditioned fear to first stimulus previously associated with shock (except control)

\begin{tabular}{lllll}
\hline $\begin{array}{l}\text { Amino } \\
\text { acid }\end{array}$ & $\begin{array}{l}\text { Noise-shift } \\
\text { Noise }\end{array}$ & $\begin{array}{l}\text { Noise-same } \\
\text { Context }\end{array}$ & $\begin{array}{l}\text { Context-same } \\
\text { Context }\end{array}$ & $\begin{array}{l}\text { Control } \\
\text { Context }\end{array}$ \\
\hline Glutamate & $200 \pm 40^{*}$ & $250 \pm 70^{*}$ & $290 \pm 90^{*}$ & $110 \pm 10$ \\
GABA & $240 \pm 60^{*}$ & $330 \pm 50^{*}$ & $380 \pm 60^{*}$ & $130 \pm 10$ \\
Aspartate & $180 \pm 40$ & $210 \pm 50^{*}$ & $260 \pm 100^{*}$ & $100 \pm 6$ \\
Taurine & $310 \pm 60^{*}$ & $330 \pm 60^{*}$ & $400 \pm 110^{*}$ & $120 \pm 15$ \\
Glycine & $330 \pm 80^{*}$ & $340 \pm 90^{*}$ & $420 \pm 100^{*}$ & $120 \pm 3$ \\
Serine & $140 \pm 80$ & $140 \pm 10$ & $140 \pm 20$ & $110 \pm 3$ \\
\hline
\end{tabular}

${ }^{*} P<0.05$, compared with basal levels.

\section{Discussion}

Rapid and transient increases in amino acid levels, including glutamate and GABA, were observed in the basolateral amygdala during the acquisition and expression of conditioned fear. The ability to make fast temporal resolution measurements allowed the demonstration of plasticity of amino acid release to discrete events during fear conditioning. To our surprise, different patterns of glutamate and GABA signaling were observed during conditioning with signaled vs. unsignaled shocks. However, glutamate and GABA showed similar changes during the expression of conditioned fear to either auditory or contextual stimuli previously associated with shock. The amino acid signals were dissociable from conditional freezing behavior during the acquisition of conditioned fear.

The most striking finding of the present study is that the nature and time course of amino acid release in the amygdala was strongly

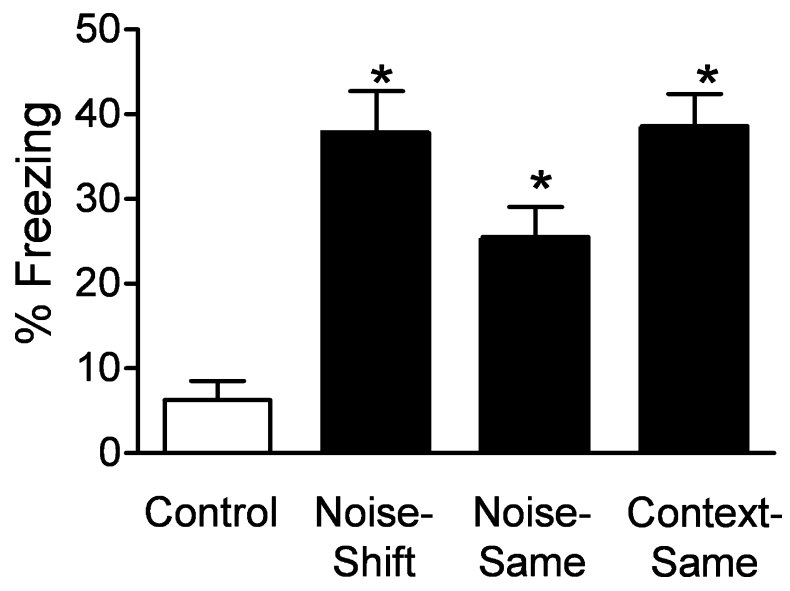

FIG. 6. Behavioral reactions for conditioned fear expression experiment. Open bars are behavioral reactions to a neutral stimulus (not previously paired with shock), and shaded bars are reactions to the first stimulus presentation previously paired with shocks. The low percentage of freezing for the control animals indicates that animals were normally active after the context shift to a neutral environment. The noise-shift animals showed significant increases in freezing behavior after the white noise CS, while the noise-same and contextsame animals showed significant increases in freezing after placement in a context previously paired with shock. A one-way ANOVA showed a main effect of group $\left(F_{3}=15.49, P<0.001\right)$, and Tukey post-test comparing individual groups showed all experimental groups were significantly greater than control $(* P<0.05)$.

dependent on whether footshock was signaled by an auditory stimulus (as in cue conditioning) or was unsignaled (as in context conditioning). On the first conditioning trial (noise-shock pairing), signaled 
shock produced a large, yet transient, increase in several amino acids, including glutamate and GABA. This quickly waned during the course of conditioning, however, as subsequent noise-shock pairings had little effect. In contrast, unsignaled shock produced a smaller increase in glutamate release on the first conditioning trial (and virtually no change in GABA release), but the magnitude of these transients tended to be sustained, or even increased, across the conditioning session. Although the basolateral amygdala is required for acquisition of both contextual and auditory cue fear memories (Goosens \& Maren, 2001), the present data suggest that the neurochemical events that accompany these two forms of fear learning are very different. Indeed, a salient auditory stimulus delivered immediately prior to footshock engenders a quantitatively and qualitatively different amino acid response in the basolateral amygdala compared with delivery of either the auditory stimulus or footshock alone. One possible explanation is that auditory afferents increase the excitability of GABAergic and glutamatergic neurons in the amygdala (Maren \& Quirk, 2004), thereby priming the response of these neurons to the footshock US.

The changes in the amino acid release after the first conditioning trial might be accounted for by two factors. First, the GABA response was minimal after unsignaled shock, which could allow sustained excitability in the amygdaloid circuit during the conditioning session, thus accounting for the progressive increase in other amino acids. In contrast, the first signaled shock was accompanied by a large increase in GABA, which may have inhibited the subsequent release of amino acids. Second, the sustained amino acid signals after unsignaled shock might represent release driven by the contextual cues associated with shock, which we have shown drive amino acid release during the expression of conditioned fear. Formal models of learning predict that unsignaled shocks would yield greater conditioning to contextual cues than signaled shock (Rescorla \& Wagner, 1972), and our data are consistent with this possibility. Indeed, the context in which unsignaled shock was delivered yielded a more robust amino acid response than that associated with signaled shock (compare Fig. 5B and $\mathrm{C}$ ). Hence, during conditioning, the salient white noise CS likely overshadowed the context in the signaled shock group and attenuated the degree to which the context would evoke amino acid release.

Glutamate release within the amygdala is believed to be required for the acquisition of conditioned fear (Maren et al., 1996; Blair et al., 2001), and microinjecting glutamate into the amygdala strengthens associations during conditioned taste aversion (Tucci et al., 1998). In the present study, glutamate levels were elevated by both signaled and unsignaled shock procedures, and the largest increase occurred after the first conditioning trial for conditioning with signaled shocks. This is consistent with the observation that a single conditioning trial is sufficient for fear learning using either signaled or unsignaled shock, and can even generate near-asymptotic levels of conditioning under some conditions (Fanselow, 1990; Maren, 1998, 1999; Nader et al., 2000). Further studies are necessary to examine whether the amino acid release profile in the amygdala after a single conditioning trial correlates with asymptotic behavioral performance. Nonetheless, both the signaled and unsignaled shock groups showed significant freezing in the intershock intervals, although the neurochemical changes were different. Therefore, differential regulation of amino acid release occurs in the amygdala during auditory vs. contextual fear learning that can be dissociated from behavioral outputs such as freezing. Because the patterns of freezing behavior and amino acid release are not the same, the neurotransmitter signals measured in the basolateral amygdala are not directly responsible for regulating freezing behavior.
In contrast to the pattern of release during conditioning, amino acid release during the expression of conditioned fear was similar after conditioning with signaled or unsignaled shocks. These results imply that amino acid signaling in the basolateral amygdala is involved in the expression of conditioned fear independent of how it was acquired. Rapid and transient increases in glutamate, GABA, glycine, aspartate and taurine, lasting about $2 \mathrm{~min}$, were observed. However, these transients were a learned response, and plasticity of amino acid release was only observed after stimuli that were previously paired with shock (compare Fig. 5A-C with Fig. 5D). Previous studies using conventional microdialysis had failed to detect any glutamate transients, probably because of insufficient temporal resolution (Yokoyama et al., 2005). The only other reported changes in amygdala GABA during expression of conditioned fear showed a decrease that lasted several hours (Stork et al., 2002). The transient increases measured in our study would not have been detected with the $20 \mathrm{~min}$ temporal resolution of their experiment, and the mechanisms of the dynamic vs. sustained GABA changes are likely different. Interestingly, the amino acid response to the conditioning context occluded the response to the auditory CS. This is similar to the occlusion of release to an auditory CS after the first conditioning trial in the signaled shock group. Again, it is possible that the stimulus-evoked GABA release determines the excitability of the system, limiting the subsequent glutamate release to the auditory CS. This transient GABA release could lead to the downregulation of $\mathrm{GABA}_{\mathrm{A}}$ receptor expression that has been reported (Chhatwal et al., 2005). Additional experiments examining amino acid signaling during repeated presentations of conditional stimuli, as in extinction, are warranted.

One of the advantages of the capillary electrophoresis technique is the ability to measure many compounds simultaneously. In addition to glutamate and GABA, the current assay allowed us to separate and detect four other amino acids: aspartate, glycine, taurine and serine. Similar patterns of release were observed during acquisition and expression of conditioned fear for aspartate, taurine and glycine as for glutamate. Serine levels were not significantly elevated in any condition, demonstrating that not all amino acids change. It is difficult to hypothesize the role for these other amino acids in the behavior because they have not been well studied during fear conditioning, but the presence of receptors for these amino acids in the amygdala suggests they could play a functional role (McCool \& Botting, 2000). It is also possible that the release of multiple amino acids may not be independent; for example, glutamate can cause GABA and taurine release (Del Arco \& Mora, 1999; Del Arco et al., 2000). Thus, the multiplicity of amino acid changes that occur in the amygdala during fear conditioning is an important observation that warrants future experimentation into the roles of these other amino acids.

In conclusion, we have demonstrated plasticity in amino acid neurotransmission the basolateral amygdala during the acquisition and expression of fear conditioning. The expression of fear responses to auditory and contextual cues previously paired with shock was associated with similar increases in amino acids, including glutamate and GABA. Thus, changes in amino acid transmission in the basolateral amygdala may be similarly involved in the expression of both kinds of fear memories. However, although the basolateral amygdala is required for the acquisition of both contextual and auditory fear conditioning, signaled vs. unsignaled shocks produced dramatically different patterns of amino acid release. We conclude that learning about contexts vs. cues is associated with very different alterations in amino acid neurotransmission in the amygdala, even though the expression of these memories is not. 


\section{Acknowledgements}

This research was supported by grants to S.M. (NIH R01 MH073655), T.E.R. (NIH R37 DA004294) and R.T.K. (NIH R01 NS38476). B.J.V. was supported by NIH T32 DA007268. We thank Lindsey Korepta and Suxia Du for assistance with behavioral analysis.

\section{Abbreviations}

CS, conditioned stimulus; GABA, $\gamma$-aminobutyric acid; US, unconditioned stimulus.

\section{References}

Blair, H.T., Schafe, G.E., Bauer, E.P., Rodrigues, S.M. \& LeDoux, J.E. (2001) Synaptic plasticity in the lateral amygdala: a cellular hypothesis of fear conditioning. Learn. Mem., 8, 229-242.

Bowser, M.T. \& Kennedy, R.T. (2001) In vivo monitoring of amine neurotransmitters using microdialysis with on-line capillary electrophoresis. Electrophoresis, 22, 3668-3676.

Chhatwal, J.P., Myers, K.M., Ressler, K.J. \& Davis, M. (2005) Regulation of gephyrin and $\mathrm{GABA}_{\mathrm{A}}$ receptor binding within the amygdala after fear acquisition and extinction. J. Neurosci., 25, 502-506.

Del Arco, A. \& Mora, F. (1999) Effects of endogenous glutamate on extracellular concentrations of GABA, dopamine, and dopamine metabolites in the prefrontal cortex of the freely moving rat: involvement of NMDA and AMPA/KA receptors. Neurochem. Res., 24, 1027-1035.

Del Arco, A., Segovia, G. \& Mora, F. (2000) Effects of endogenous glutamate on extracellular concentrations of taurine in striatum and nucleus accumbens of the awake rat: involvement of NMDA and AMPA/kainate receptors. Amino Acids, 19, 729-738.

Fanselow, M.S. (1990) Factors governing one-trial contextual conditioning. Anim Learn. Behav., 18, 264-270.

Fanselow, M.S. \& Kim, J.J. (1994) Acquisition of contextual Pavlovian fear conditioning is blocked by application of an NMDA receptor antagonist D, L2-amino-5-phosphonovaleric acid to the basolateral amygdala. Behav. Neurosci., 108, 210-212.

Fanselow, M.S. \& LeDoux, J.E. (1999) Why we think plasticity underlying pavlovian fear conditioning occurs in the basolateral amygdala. Neuron, 23, 229-232.

Goosens, K.A. \& Maren, S. (2001) Contextual and auditory fear conditioning are mediated by the lateral, basal, and central amygdaloid nuclei in rats. Learn. Mem., 8, 148-155.

Goosens, K.A. \& Maren, S. (2004) NMDA receptors are essential for the acquisition, but not expression, of conditional fear and associative spike firing in the lateral amygdala. Eur. J. Neurosci., 20, 537-548.

LeDoux, J.E. (2000) Emotion circuits in the brain. Ann. Rev. Neurosci., 23, $155-184$.

Maren, S. (1996) Synaptic transmission and plasticity in the amygdala - an emerging physiology of fear conditioning circuits. Mol. Neurobiol., 13, $1-22$.

Maren, S. (1998) Overtraining does not mitigate contextual fear conditioning deficits produced by neurotoxic lesions of the basolateral amygdala. J. Neurosci., 18, 3088-3097.

Maren, S. (1999) Neurotoxic basolateral amygdala lesions impair learning and memory but not the performance of conditional fear in rats. J. Neurosci., 19, 8696-8703.

Maren, S. (2001) Neurobiology of Pavlovian fear conditioning. Ann. Rev. Neurosci., 24, 897-931.
Maren, S., Aharonov, G., Stote, D.L. \& Fanselow, M.S. (1996) N-methyl-Daspartate receptors in the basolateral amygdala are required for both acquisition and expression of conditional fear in rats. Behav. Neurosci., 110, $1365-1374$.

Maren, S. \& Quirk, G.J. (2004) Neuronal signalling of fear memory. Nat. Rev. Neurosci., 5, 844-852

McCool, B.A. \& Botting, S.K. (2000) Characterization of strychnine-sensitive glycine receptors in acutely isolated adult rat basolateral amygdala neurons. Brain Res., 859, 341-351.

Miserendino, M.J., Sananes, C.B., Melia, K.R. \& Davis, M. (1990) Blocking of acquisition but not expression of conditioned fearpotentiated startle by NMDA antagonists in the amygdala. Nature, $\mathbf{3 4 5}$, 716-718.

Muller, J., Corodimas, K.P., Fridel, Z. \& LeDoux, J.E. (1997) Functional inactivation of the lateral and basal nuclei of the amygdala by muscimol infusion prevents fear conditioning to an explicit conditioned stimulus and to contextual stimuli. Behav. Neurosci., 111, 683-691.

Nader, K., Schafe, G.E. \& LeDoux, J.E. (2000) Fear memories require protein synthesis in the amygdala for reconsolidation after retrieval. Nature, 406, $722-726$.

Paez, X. \& Hernandez, L. (2001) Biomedical applications of capillary electrophoresis with laser-induced fluorescence detection. Biopharm. Drug Dispos., 22, 273-289.

Parrot, S., Bert, L., Mouly-Badina, L., Sauvinet, V., Colussi-Mas, J., LambasSenas, L., Robert, F., Bouilloux, J.P., Suaud-Chagny, M.F., Denoroy, L. \& Renaud, B. (2003) Microdialysis monitoring of catecholamines and excitatory amino acids in the rat and mouse brain: recent developments based on capillary electrophoresis with laser-induced fluorescence detectiona mini-review. Cell. Mol. Neurobiol., 23, 793-804.

Paxinos, G. \& Watson, C. (1998) The Rat Brain in Stereotaxic Coordinates. Academic Press, New York.

Rescorla, R.A. \& Wagner, A.R. (1972) A Theory of Pavlovian Conditioning: Variations in the Effectiveness of Reinforcement and Nonreinforcement. Appleton-Century-Crofts, New York.

Shackman, J.G., Watson, C.J. \& Kennedy, R.T. (2004) High-throughput automated post-processing of separation data. J. Chrom. A, 1040, 273-282.

Stork, O., Ji, F.Y. \& Obata, K. (2002) Reduction of extracellular GABA in the mouse amygdala during and following confrontation with a conditioned fear stimulus. Neurosci. Lett., 327, 138-142.

Tucci, S., Rada, P. \& Hernandez, L. (1998) Role of glutamate in the amygdala and lateral hypothalamus in conditioned taste aversion. Brain Res., 813, 44-49.

Venton, B.J., Robinson, T.E. \& Kennedy, R.T. (2006) Transient changes in nucleus accumbens amino acid concentrations correlate with individual responsivity to the predator fox odor 2,5-dihydro-2,4,5-trimethylthiazoline. J. Neurochem., 96, 236-246.

Walker, D.L. \& Davis, M. (2002) The role of amygdala glutamate receptors in fear learning, fear-potentiated startle, and extinction. Pharm. Biochem. Behav., 71, 379-392.

Wilensky, A.E., Schafe, G.E. \& LeDoux, J.E. (1999) Functional inactivation of the amygdala before but not after auditory fear conditioning prevents memory formation. J. Neurosci., 19, RC48, 1-5.

Yokoyama, M., Suzuki, E., Sato, T., Maruta, S., Watanabe, S. \& Miyaoka, H. (2005) Amygdalic levels of dopamine and serotonin rise upon exposure to conditioned fear stress without elevation of glutamate. Neurosci. Lett., 379, $37-41$.

Young, A.M.J. (2004) Increased extracellular dopamine in nucleus accumbens in response to unconditioned and conditioned aversive stimuli: studies using $1 \mathrm{~min}$ microdialysis in rats. J. Neurosci. Meth., 138, 57-63. 